\title{
Nocardia Co-Infection in Patients With Pulmonary Tuberculosis
}

\author{
Alireza Ekrami ${ }^{1,2, *}$; Azar Dokht Khosravi ${ }^{2,3}$; Ali Reza Samarbaf-Zadeh ${ }^{2,3}$; Mohammad \\ Hashemzadeh $^{3}$ \\ ${ }^{1}$ Department of Medical Laboratory Sciences, School of Paramedicine, Ahvaz Jundishapur University of Medical Sciences, Ahvaz, IR Iran \\ 2 Infectious and Tropical Diseases Research center, Ahvaz Jundishapur University of Medical Sciences, Ahvaz, IR Iran \\ ${ }^{3}$ Department of Microbiology, School of Medicine, Ahvaz Jundishapur University of Medical Sciences, Ahvaz, IR Iran \\ *Corresponding author: Alireza Ekrami, Department of Medical Laboratory Sciences, School of Paramedicine, Ahvaz Jundishapur University of Medical Sciences, Ahvaz, IR Iran. Tel: \\ +98-6113837317, Fax:+98-6113738330, E-mail: aekrami@yahoo.com, ekrami@ajums.ac.ir
}

Received: July 2, 2013; Revised: January 14, 2014; Accepted: January 18, 2014

\begin{abstract}
Background: Tuberculosis (TB) remains as one of the most serious infectious diseases in the world. Pulmonary tuberculosis can occur with other pulmonary diseases caused by opportunistic organisms such as Nocardia spp. particularly in immunocompromised patients. Therefore, diagnosis of co-infection at the early stage of the disease could be lifesaving.

Objectives: The goal of this study was to detect Mycobacterium tuberculosis and Nocardia spp. in sputum specimens in order to assess the concomitant nocardiosis and tuberculosis in patients with suspected pulmonary tuberculosis.

Patients and Methods: From March 2011 to April 2012,189 sputum specimens were obtained from patients who were suspected of having pulmonary tuberculosis. Out of 189 samples, 32 of the samples belonged to hospitalized HIV-infected patients. Samples were examined by Gram and Ziehl-Nelsen staining, culture and PCR methods.

Results: From 157 sputum specimens, positive samples by acid fast staining, culture and PCR for M. tuberculosis were reported for 7.6\% (12/157), 10.1\% (16/157) and 7\% (11/157) of samples, respectively. No results were obtained by the described methods for Nocardia spp. Among 32 samples of HIV-infected patients, four (12.5\%) had positive results for acid fast staining, culture and PCR detecting M. tuberculosis while only two samples had positive results for Nocardia spp. by PCRand no results were reported by culture, Gram and acid fast staining for this organism.

Conclusions:Concurrent pulmonary nocardiosis and tuberculosis is frequent in HIV-infected patients. Rapid and sensitive methods such as PCR are recommended for detection of such co-infections.
\end{abstract}

Keywords:Mycobacterium tuberculosis; Nocardia; Pulmonary tuberculosis; HIV

\section{Background}

Tuberculosis (TB) is caused by the Mycobacterium tuberculosis, a member of the M. tuberculosis complex (MTBC). According to the World Health Organization (WHO), in 2010, there were an estimated 12.0 million prevalent cases of TB (equivalent to 178 cases per 100000 individuals) and approximately 1.4 million people died of TB during that year. Most of the cases in 2009 (55\%) occurred in Asia (1). According to the "Administration of Tuberculosis and Leprosy Control" of the Ministry of Health and Medical Education in Iran, in 2010, a total of 10485 old and new cases of TB were reported in Iran and of these cases, 326 patients (around 2.2\%) were HIV positive (2). The diseases caused by Mycobacterium complex have become more important in the recent years, particularly in association with the worldwide pandemic situation caused by $\mathrm{Hu}-$ man Immunodeficiency Virus (HIV). In 2010, an estimated 1.1 million deaths occurred among HIV-negative women with TB. This was equivalent to 15 deaths per 100000 individuals. In addition, there were an estimated 0.35 million deaths among TB cases that were HIV-positive. Ac- cording to WHO, the incidence of pulmonary tuberculosis in HIV-positive patients in Iran was 0.38 (0.28-0.51) per 100000 individuals in 2012 (3).

Some opportunistic microorganisms such as Nocardia spp.can mimic pulmonary tuberculosis particularly in immunocompromised patients. Nocardia spp. are Gram-positive (weakly acid fast) and filamentous with a branching appearance and are not part of the normal human bacterial flora. To date at least sixteen species capable of producing disease in humans have been identified (4). Pulmonary disease is the predominant clinical presentation of this microorganism and can be fatal if untreated. Untreated pulmonary nocardiosis is similar to tuberculosis and N. asteroids is the most frequent cause of pulmonary infection in humans (85\%) (5). Since the clinical and radiological manifestations are non-specific, pulmonary nocardiosis could be mistaken with other infections or other bacterial pneumonia. In addition, nocardiosis may be under reported because it is not among the AIDS-defining criteria (6). Diagnosis of pulmonary nocardiosis is dependent on

Copyright (C) 2014,Ahvaz Jundishapur University of Medical Sciences; Published by Kowsar. This is an open-access article distributed under the terms of the Creative Commons Attribution-NonCommercial 4.0 International License (http://creativecommons.org/licenses/by-nc/4.0/) which permits copy and redistribute the material just in noncommercial usages, provided the original work is properly cited. 
the isolation or demonstration of the organism from respiratory secretions such as sputum or tissue specimens. Nocardia as a M. tuberculosis is a slow growing organism that requires prolonged incubation for at least 4-6 weeks (7). The diagnosis of nocardiosis is currently based on direct examination and conventional culture; serology is usually not useful. Treatment of nocardiosis is different from tuberculosis regarding therapeutic agents, route of administration and duration of therapy (8). Sulfonamides have been the agents of choice for the treatment of nocardiosis, however combination therapy with two or more agents is recommended. Although nocardiosis is an uncommon illness, co-infection of this disease with tuberculosis has been reported (9).

\section{Objectives}

The aim of this study was to assess the presence of Nocardia spp. and M. tuberculosis in sputum specimens of patients with pulmonary tuberculosis. In the meantime, 32 HIV-infected patients who were suspected of having pulmonary tuberculosis were included in this study in order to evaluate concomitant infection in these patients.

\section{Patients and Methods}

\subsection{Sputum Collection and Processing}

A total of 189 sputum samples were obtained from the reference mycobacteriology laboratory, Ahvaz Jundishapur University of Medical Sciences from March 2011 to April 2012. All specimens were obtained from patients with suspected pulmonary tuberculosis. Among 189 specimens, 32 were collected from hospitalized HIV-positive patients. All specimens were processed immediately according to standard routine diagnosis procedures by the $\mathrm{NaOH} / \mathrm{N}$-Acetyl-L-cysteine (NALC) method. Next, concentrated specimens were used for smear preparation, culture and PCR assay $(10,11)$. Furthermore, $250 \mu \mathrm{L}$ of each concentrated sputum specimen was inoculated onto Lowenstein-Jensen (L-J) media (Merck, Germany). All cultures were incubated at $37^{\circ} \mathrm{C}$ with $5 \% \mathrm{CO}_{2}$ and humidity. Nocardia spp. are acid fast and can survive the decontamination of clinical specimens with sodium hydroxide and NALC methods, thereby, they can grow on L-J medium. Suspicious colonies were examined with acid-fast and Gram staining methods. Cultures were examined weekly and after 8 weeks of incubation, cultures, which showed no growth, were reported negative and discarded. For microscopic examination, using a disposable Pasteur pipette, one drop of the sediment of the sputum specimen was spread on each clean microscope slide and after air drying and fixation by heat, the smears were stained by Ziehl-Neelsen and Gram staining (10).

\subsection{DNA Extraction and PCR Assay}

After decontamination, the chromosomal DNA was ex- tracted using proteinase $\mathrm{K}$ and phenol:chloroform, and then precipitated by ethanol and isopropanol, according to standard procedures (12). The purified DNA was amplified with specific pairs of primers IS1 (5'CTCGTCCAGCGCCGCTTCGG 3') and IS2 (5'CCTGCGAGCGTAGGCGGTGG 3') for $M$. tuberculosis complex strains Ann Microbiol. The PCR protocols were optimized to 35 cycles consisting of 45 seconds at $94^{\circ} \mathrm{C}$ for denaturation, 30 seconds at $68^{\circ} \mathrm{C}$ for annealing and 30 seconds at $72^{\circ} \mathrm{C}$ for extension, followed by a final 5 minute extension at $72^{\circ} \mathrm{C}$.

Primers NG1 (5'CTCGTCCAGCGCCGCTTCGG3') and NG2 (5'CCTGCGAGCGTAGGCGGTGG3') were used to amplify $a$ Nocardia genus specific 590-bp fragment of 165 rRNA. The PCR protocols were optimized to 40 cycles consisting of 45 seconds at $94^{\circ} \mathrm{C}$ for denaturation, 30 seconds at $57^{\circ} \mathrm{C}$ for annealing and 30 seconds at $72^{\circ} \mathrm{C}$ for extension, followed by a final 5 minute extension at $72^{\circ} \mathrm{C}$. Both reactions were performed in a final volume of $25 \mu \mathrm{L}$. PCR was performed as described by Eisenach et al. and Couble et al. respectively $(13,14)$. Primers oligonucleotides for PCR were purchased from TaG Co. (Copenhagen-Denmark).

Amplification with these primers was observed by electrophoresis on $2 \%(\mathrm{w} / \mathrm{v})$ agarose gel stained with ethidium bromide (Figure 1). The N. asteroides ATCC 19247 and M. tuberculosis H37RV were used as positive reference strains. Two PCRs for each sample were performed in separate tubes with two pairs of primers. One set was dedicated to the $M$. tuberculosis complex, whereas the other set was for Nocardia spp. For each round of PCR, $\mathrm{ddH}_{2} \mathrm{O}$ was used as the negative template. The investigators were blinded to clinical data during the experiment for detection of microbial agents' co-infection. All specimens were unnamed and labeled by a secure code in the reference mycobacteriology laboratory.

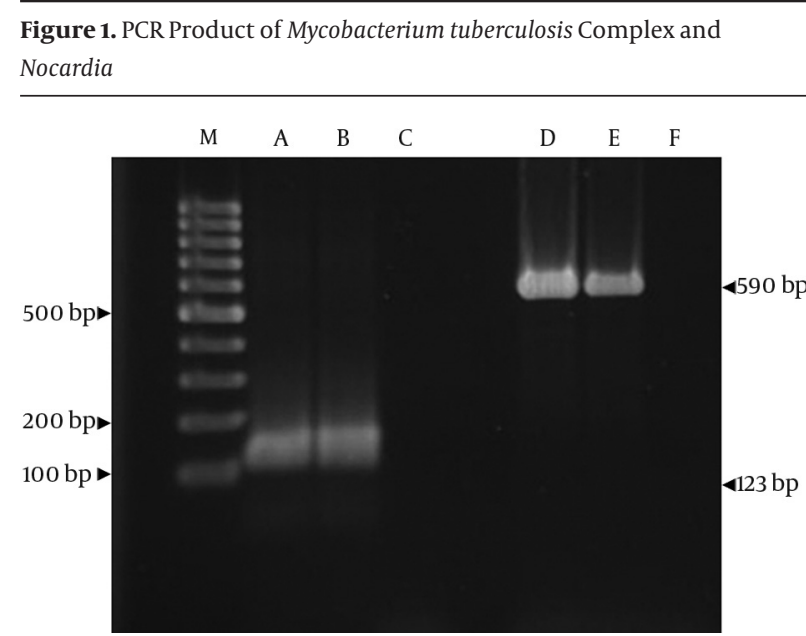

A: Clinical sample of M. tuberculosis. B and C: Positive and negative controls. D: Clinical sample of Nocardia. E and F: Positive and negative controls. M; 100 bp DNA size marker 


\section{Results}

\subsection{Specimens}

Among the 157 outpatients, 7.6\% (12/157) of specimens had positive results for acid fast bacilli by acid fast staining but none of the samples were positive for Nocardia spp. After cultures evolution, $10.1 \%$ (16/157) of specimens had positive results for MTBC while no specimens grew Nocardia spp. During microscopic examination by the Gram staining method, we couldn't find any particle morphologically similar to Nocardia. Out of 157 samples, 7\% (11/157) were positive by PCR for MTBC and none of the samples was positive for Nocardia by PCR. Among 32 samples in HIV-infected patients, four (12.5\%) were culture positive for M. tuberculosis and none of them were positive for Nocardia. Furthermore, these four samples were positive by acid fast staining and PCR assay. Out of these four samples, two were positive for Nocardia spp. by the PCR method. Based on our data the prevalence of nocardiosis and also concomitant infection with tuberculosis in our study was 6.25\% (2/32) in HIV-infected patients and $1.05 \%$ (2/189) for all cases.

\section{Discussion}

As clinical manifestation of pulmonary tuberculosis and nocardiosis are similar, laboratory tests are required in order to distinguish of nocardiosis from tuberculosis. Nocardia is a slow growing bacteria and the contamination risk of the culture with other bacteria and fungi is high. Therefore, the suggested approach for this problem is molecular techniques, since they are more sensitive than conventional diagnostic methods (15). As two samples were positive for Nocardia spp. and MTBC using the PCR method, it is suggested that concurrent pulmonary tuberculosis and nocardiosis may developed coincidently. Nocardiosis has been reported to be more prevalent in HIV infected patients. In these patients, the incidence of nocardiosis is approximately 140 folds higher than in the general population. Infection with opportunistic agents such as Nocardia is attributed to suppression of cell mediated immunity (16).

It has been suggested that, the most common condition predisposing patients to nocardiosis is underlying chronic lung disease. In this study it was found that the coincidence of pulmonary tuberculosis and nocardiosis was $1 \%$ for the entire study population and $6.25 \%$ among HIV-infected patients. The incidence of pulmonary nocardiosis in HIV-infected patients has been previously reported by some investigators. Pulmonary nocardiosis in HIV-infected patients with suspected pulmonary tuberculosis was reported to be $3 \%$ by Alnaum et al.; they reported an average of 3-4\% for this infection (17). Some reports indicate that greater than two-thirds of the patients diagnosed with pulmonary nocardiosis were initially diagnosed as having tuberculosis and about $5 \%$ of the patients with proven pulmonary tuberculosis were shown to have co-infection with Nocardia (18).
Out of 140 sputum samples from African HIV-positive individuals clinically suspected of having tuberculosis (TB), the frequency of nocardiosis was reported as 3.6\% (19). In areas where HIV-associated TB is common, some patients diagnosed as smear negative pulmonary tuberculosis, might also suffer from nocardiosis. Only a few case reports of concomitant infection of Nocardia and TB have been published in the literature (20). A prevalence of $1.4 \%$ for pulmonary nocardiosis was reported in a tuberculosis and chest diseases hospital in Amritsar by Singh et al. (21). Although, Pintado et al. indicated that the overall incidence of nocardiosis among HIVinfected patients is between 0.1 and $0.4 \%$, and is associated with high morbidity and mortality rates (18). Concurrent infection of pulmonary nocardiosis with other microorganisms is not rare. Concurrent pulmonary aspergillosis and nocardiosis was reported in an immunocompromised patient following long-term steroid therapy (22).

In this study Nocardia was not distinguished in sputum specimens using conventional methods, however, positive samples were determined using the PCR assay. Our study showed that the PCR technique was more sensitive than conventional methods in detection of Nocardia. Accordance of molecular techniques with conventional methods has been reported as 70 to $90 \%$ by several studies $(22,23)$.

In Iran, several studies investigated pulmonary nocardiosis or tuberculosis in patients with various clinical symptoms. Recently, Aminzade et al. reported concomitant pulmonary nocardiosis and tuberculosis in a patient with rheumatoid arthritis (24). In a study by Eshraghi and Amin, Nocardia asteroides was isolated from only one patient suffering from Cushing's syndrome with bronchogenic carcinoma amongst 142 patients with advanced symptomatic pulmonary disease in Tehran, Iran (25). In another study from Iran (Shojaei et al.) clinical isolation of $N$. cyricigeorgica from patients with various clinical manifestations was performed (26). To the best of our knowledge, this is the first study reporting co-infection of nocardiosis and tuberculosis in HIV-positive patients in Iran.

Concurrent Pulmonary tuberculosis and nocardiosis is much more frequent in HIV-infected patients and can be fatal. Although nocardiosis resembles tuberculosis, first line anti-tuberculous drugs are not efficient for its treatment. Therefore, it is important to establish a potent diagnosis method with high sensitivity and specificity such as molecular methods to improve the speed of diagnosis of nocardiosis.

\section{Acknowledgements}

We gratefully thank members of the reference mycobacteriology laboratory for their help.

\section{Authors' Contributions}


Study design: Dr. Alireza Ekrami and Dr Azar Dokht Khosravi. Drafting of the manuscript: Dr. Ali Reza Samarbaf Zadeh and Mr. Mohammad Hashemzadeh.

\section{Funding/Support}

This study was approved by the Infectious and Tropical Diseases Research Center and supported by a grant (No. 88106) from the Research Affairs of Ahvaz Jundishapur University of Medical Sciences.

\section{References}

1. World Health Organization . Global tuberculosis control. Geneva; 2012.

2. Neibecker M, Schwarze-Zander C, Rockstroh JK, Spengler U, Blackard JT. Evidence for extensive genotypic diversity and recombination of GB virus C (GBV-C) in Germany. J Med Virol. 2011;83(4):685-94.

3. World Health Organization . WHO policy on collaborative TB/HIV activities: guidelines for national programmes and other stakeholders. Geneva; 2012

4. McPhee SJ, Papadakis MA, Rabow MW. Current medical diagnosis \& treatment 2010.: McGraw-Hill Medical; 2010.

5. Martinez R, Reyes S, Menendez R. Pulmonary nocardiosis: risk factors, clinical features, diagnosis and prognosis. Curr Opin Pulm Med. 2008;14(3):219-27.

6. Nwuba C, Ogbu N, Okonkwo R, Abolarin O, Modebelu P. Nocardiosis-an emerging complication in the clinical management of HIV/AIDS patients in Africa. J Int Aids Soc. 2012;15(6).

7. Gupta D, Dutta G. Pulmonary Nocardiosis Mimicking Tuberculosis-A Case Report. Int J Contemp Med. 2013;1(1):24-8.

8. Tremblay J, Thibert L, Alarie I, Valiquette L, Pepin J. Nocardiosis in Quebec, Canada, 1988-2008. Clin Microbiol Infect. 2011;17(5):690-6.

9. Welsh O, Vera-Cabrera L, Salinas-Carmona MC. Current treatment for nocardia infections. Expert Opin Pharmacother. 2013;14(17):2387-98.

10. World Health Organization . Laboratory services in TB control, Part II: Microscopy. Geneva; 1998.

11. Read RC. Nocardiosis and actinomycosis. Medicine. 2009; 37(12):657-9.

12. Amita J, Vandana T, Guleria R, Verma R. Qualitative evaluation of mycobacterial DNA extraction protocols for polymerase chain reaction. Mol Biol Today. 2002;3:43-50.
13. Eisenach KD, Cave MD, Bates JH, Crawford JT. Polymerase chain reaction amplification of a repetitive DNA sequence specific for Mycobacterium tuberculosis. J Infect Dis. 1990;161(5):977-81.

14. Couble A, Rodriguez-Nava V, de Montclos MP, Boiron P, Laurent F Direct detection of Nocardia spp. in clinical samples by a rapid molecular method. J Clin Microbiol. 2005;43(4):1921-4.

15. Brown-Elliott BA, Brown JM, Conville PS, Wallace RJ. Clinical and laboratory features of the Nocardia spp. based on current molecular taxonomy. Clin Microbiol Rev. 2006;19(2):259-82.

16. Filice GA. Nocardiosis in persons with human immunodeficiency virus infection, transplant recipients, and large, geographically defined populations. J Lab Clin Med. 2005;145(3):156-62.

17. Alnaum HM, Elhassan MM, Mustafa FY, Hamid ME. Prevalence of Nocardia species among HIV-positive patients with suspected tuberculosis. Trop Doct. 2011;41(4):224-6.

18. Pintado V, Gomez-Mampaso E, Cobo J, Quereda C, Meseguer MA, Fortun J, et al. Nocardial infection in patients infected with the human immunodeficiency virus. Clin Microbiol Infect. 2003;9(7):716-20.

19. Rasheed MU, Belay G. Nocardiosis in HIV seropositive clinically suspected pulmonary tuberculosis patients. Trop Doct. 2008;38(1):34-5.

20. Chaudhury RC, Aher AR, Rastogi V, Prabhu TK. A case of mixed pulmonary infection by nocardia and Mycobacterium tuberculosis. Indian J Pathol Microbiol. 2009;52(2):294-5.

21. Singh M, Sandhu RS, Randhawa HS, Kallan BM. Prevalence of pulmonary nocardiosis in a tuberculosis hospital in Amritsar, Punjab. Indian J Chest Dis Allied Sci. 2000;42(4):325-39.

22. Mohan A, Sharma SK, Arora VK, Sharma S, Prakash J. Concurrent pulmonary Aspergillosis and Nocardiosis in an old tubercular cavity masquerading as malignancy in an immunocompetent individual. Resp Med. 2008;1(3):231-4.

23. Ambrosioni J, Lew D, Garbino J. Nocardiosis: updated clinical review and experience at a tertiary center. Infection. 2010; 38(2):89-97.

24. Aminzadeh Z, Alavi Darazam I. Concomitant Pulmonary Nocardiosis and Tuberculosis in a Patient With Rheumatoid Arthritis: A New View Point. Jundishapur J Microbiol. 2012;6(1):91-4.

25. Eshraghi S, Amin M. Nocardia asteroides Complex in Patient with Symptomatic Pulmonary Nocardiosis in a patient with bronchiectasis. Iran J Public Health.2001;30(3-4):99-102.

26. Shojaei H, Hashemi A, Heidarieh P, Eshraghi S, Khosravi AR, Daei Naser A. Clinical isolation of Nocardia cyriacigeorgica from patients with various clinical manifestations, the first report from Iran. Med Mycol J. 2011;52(1):39-43. 